\title{
Characterization of Mesenchymal Stem Cells from Human Cortical Bone
}

\author{
Joseph S. Fernandez-Moure ${ }^{1, *}$, Bruna Corradetti ${ }^{2}$, Trevor Janecek ${ }^{1}$, \\ Jeffrey Van Eps ${ }^{1}$, Matthew Burn ${ }^{1}$, Bradley K. Weiner ${ }^{1}$, \\ Pranela Rameshwar ${ }^{3}$ and Ennio Tasciotti ${ }^{2}$
}

${ }^{1}$ The Methodist Hospital Research Institute, Department of Surgery,
Houston Methodist Hospital, Houston, Texas, USA
${ }^{2}$ Houston Methodist Research Institue, Houston, Texas, USA
${ }^{3}$ Rutgers New Jersey Medical School, Newark, New Jersey, USA
${ }^{*}$ Corresponding Author: jsfernandez-moure@houstonmethodist.org

Received 1 July 2016; Accepted 1 October 2016;

Publication 21 November 2016

\section{Abstract}

Background Context: Mesenchymal stem cells (MSC) are being used for spine and orthopaedic surgical and research applications. Bone marrow and fat are the most commonly used sources of these cells.

Purpose: To describe a new technique allowing the isolation and expansion of human MSC from cortical bone.

Study Design: MSC from human cortical bone (vertebral lamina) were isolated, expanded, and verified in vitro.

Methods: Human MSC were isolated from laminar bone obtained during surgery (decompression/laminectomy). They were then cultured and assessed using fluorescence-activated cell sorting techniques for MSC markers, colonyforming unit assays, and multilineage differentiation.

Results: Isolated and cultured cells demonstrated MSC markers and trilineage differentiation confirming their stemness.

International Journal of Translational Science, Vol. 2016, 71-86.

doi: 10.13052/ijts2246-8765.2016.005

(c) 2016 River Publishers. All rights reserved. 
Conclusion: A novel method for the isolation of MSC from cortical bone has been described. These cells have significant current and future application in spine and orthopaedic surgery; and both the source of the cells and particular characteristics of the cortical bone derived MSC have advantages over currently used MSC obtained from other sources.

\section{Introduction}

Mesenchymal stem cells (MSC) are currently a primary focus in spine and orthopaedic surgery as well as regenerative medicine. Derived from mesenchyme - cells of mesoderm origin in human embryos - they are capable of differentiating into bone, cartilage [1], fat [2] tendon [3], and muscle [4]; and thus have obvious appeal for applications in musculoskeletal repair and tissue engineering including spinal surgery where they might be used to regenerate or replace degenerating tissues and enhance spinal arthrodesis.

MSC have been retrieved from a variety of tissues from throughout the body including periosteum [5], muscle [4], circulating blood [6], blood vessels [7], synovium [8], bone marrow [9], fat [10], and trabecular bone [11, 12]. Currently, bone marrow and fat are the most commonly used sources in humans in spinal surgery, however the yield is often poor and their efficacy for osteogenesis or regenerative purposes is still unclear. For the purposes of osteogenic regeneration, MSCs isolated from the cortical fraction of spinal lamina, have been shown to have greater osetogenic function when compared to cells isolated from adipose or bone marrow [11]. Yet, a comprehensive characterization of compact bone MSCs isolated from human spinal lamina has yet to be performed.

MSCs retrieval from cortical bone of mice has been described [12], however to date, cortical bone has not been described as a source of multipotent MSC cells with regenerative capabilities in humans. In this paper, we describe a method for isolation and characterization of MSC from a previously undescribed source, the adult human cortical bone.

\section{Materials and Methods}

\subsection{Isolation of Human Cortical Bone Mesenchymal Stem Cells}

Following laminectomy of the lower lumbar spine, bone fragments obtained were cleaned by sharp dissection of any fat, connective tissue, or muscle. Any blood that remained on the specimen was flushed from the bony fragments with phosphate buffered saline (PBS) (ThermoScientific) with $1 \%$ 
antibiotic/antimycotic (Gibco, USA) until none could be seen and they appeared clean of all debris. Fragments were then crushed into chips approximately 3-4 $\mathrm{mm}^{3}$ and transferred to $50 \mathrm{ml}$ polypropylene tubes (BD Falcon, Bedford, MA, USA). The bone chips were suspended in $\alpha$-MEM containing $2 \%(\mathrm{v} / \mathrm{v})$ defined fetal bovine serum (FBS) (Gibco, USA) in the presence of $3 \mathrm{mg} / \mathrm{mL}$ collagase type-I (Worthington Biochemical Corp., 130 Freehold, NJ) and $4 \mathrm{mg} / \mathrm{mL}$ dispase II (Roche, Indianapolis, IN) and placed on a shaking platform at $37^{\circ} \mathrm{C}$ for three hours. This was then strained through a $70 \mu \mathrm{m}$ filter into another $50 \mathrm{~mL}$ polypropylene tube to separate the bone fragments. Any remaining bone debris that was not removed was allowed to settle to the bottom of the tube for $10 \mathrm{~min}$ and the supernatant collected. The supernatant was then centrifuged at $400 \mathrm{~g}$ for $5 \mathrm{~min}$ at $4^{\circ} \mathrm{C}$. This was repeated to ensure maximal cell recovery and removal of bone debris. The cells were then plated in plastic culture flasks in $\alpha$-MEM containing $20 \%$ (v/v) FBS and incubated at $37^{\circ} \mathrm{C}$ in a $5 \% \mathrm{CO}_{2}$ incubator. After 48 hours the media was changed to remove all non-adherent cells.

\subsection{Culture of Isolated Compact Bone-Derived Mesenchymal Stem Cells}

Adherent cells were cultured in $\alpha$-MEM containing 20\% (vol/vol) FBS supplemented with $1 \%$ antibiotic/antimycotic (Gibco, USA) and incubated at $37^{\circ} \mathrm{C}$ in a $5 \% \mathrm{CO}_{2}$ incubator. The media was changed every 48 hours until the cells were at 60-80\% confluency at which point they were passaged at a split ratio of 1:3. Cells in passage 4-6 were used in all subsequent characterizations in this study.

\subsection{Histological Staining of Bone Fragments}

To assess to complete removal of cells from the bone matrix histologic staining was performed. Bone fragments underwent digestion as mentioned above. Prior to, and after complete digestion fragments were fixed in $10 \%$ buffered formalin and then embedded in paraffin. Tissue samples were then cut into $4 \mu \mathrm{m}$ sections using a microtome and decalcified. Three non-consecutive sections were routinely stained with Haemotoxylin and Eosin (H\&E) and inspected for presence of cells following digestion.

\subsection{Fluorescence-Activated Cell Sorting}

Once a sufficient cell number was achieved, mesenchymal stem cells were selected by using fluorescence activated assisted cell sorting (FACS) system 
based on a panel of lineage committed cell surface markers Adherent cells were harvested using TypLE ${ }^{\mathrm{TM}}$ Express (Gibco, USA), washed with cold PBS and then selected two separate cell sorts to isolate the final fraction of cells of interest. In order to fully characterize the cells for 9 markers two separate sorts were required. Briefly, $1 \times 10^{7}$ cells were harvested, suspended in staining buffer (PBS $+3 \% \mathrm{FBS}$ ), and incubated with fluorescent antibodies to the previously mentioned cell surface markers and incubated at $4{ }^{\circ} \mathrm{C}$ in the dark for $30 \mathrm{~min}$. Unstained cells were used as negative controls. The antibody combination for the first portion of the experiment was CD34/PECy5.5 (Invitrogen, Burlington, Ont, Canada), CD45/APC-Cy7 (Biolegend, San Diego, Ca), CD29/PE (Biolegend, San Diego, Ca), CD73/FITC (Biolegend, San Diego, Ca), CD105/APC (Invitrogen, Burlington, Ont, Canada). The sorted cells were then re-plated in standard media and allowed to expand for one week. Following this incubation period they were then harvested as previously mentioned, washed, and stained using a second set of antibodies. The antibody combination for the second portion was CD44/FITC (Invitrogen, Burlington, Ont, Canada), HLA-DR/PE-Cy5 (Biolegend, San Diego, Ca), CD19/APC (eBioscience, San Diego, Ca), CD90/PE (Biolegend, San Diego, Ca).

Acquisition was performed on a FACS using a BD FACS Aria II (Becton Dickinson, Franklin Lakes, NJ) and BD FACS Diva software (Becton Dickin-

son, Franklin Lakes, NJ) was used for data analysis. Sorted cells were collected and cultures were established in HG-DMEM with $10 \%$ FBS and then plated onto plastic culture flasks.

\subsection{Colony Forming Unit (CFU) Assay}

$\mathrm{CFU}$ assays were performed at $\mathrm{P} 0$ on freshly isolated cells $(\mathrm{n}=3)$ at different densities $\left(100,250,500\right.$, and 1000 cells $\left./ \mathrm{cm}^{2}\right)$. Cells were plated in six-well plates and cultured in $5 \% \mathrm{CO}_{2}$ and $90 \%$ humidity at $37^{\circ} \mathrm{C}$ for 2 weeks in HGDMEM-supplemented medium. Then, colonies were fixed with $4 \%$ formalin and stained with $1 \%$ toluidin blue solution (Fluka BioChemika, Buchs, Switzerland) at room temperature for 2 hours, and washed twice. Colonies formed by 16-20 nucleated cells were counted under a BX71 microscope (Olympus).

\subsection{Multilineage Differentiation}

To assess the multilineage differentiation capacity of these cells, in vitro osteogenic, adipogenic, and chondrogenic inductions were performed. 
For osteogenic differentiation, passage 2-4 MSCs were seeded into a 12well plate at a density of $5 \times 10^{3}$ cells $/ \mathrm{cm}^{2}$. Cells were incubated at $37^{\circ} \mathrm{C}$ in a humidified atmosphere of $5 \% \mathrm{CO}_{2}$ for 24 hours in standard media. Following this incubation the media was replaced with osteogenic differentiation media (Gibco) and cells were allowed to undergo differentiation for 14 days with media change every 3 days. Mineral deposits were then highlighted by Von Kossa staining.

To assess chondrogenic differentiation micromass cultures were generated by seeding $5-\mu 1$ droplets of a $1.6 \times 10^{7}$ cells $/ \mathrm{ml}$ suspension into the center of a multi-well culture vessel. The micromass was allowed to cultivate for 2 hours at $37^{\circ} \mathrm{C}$ in a humidified atmosphere of $5 \% \mathrm{CO}_{2}$ after which complete chondrogenic differentiation media (Gibco) was gently added so as to not perturb the micromass. Cells were allowed to undergo differentiation for 21 days with media change every 2 days. Following induction, glucosaminoglycans and mucopolyscacharides produced by the cells were stained by Alcian blue.

Cells undergoing adipogenic differentiation were seeded into culture vessels at a density of $1 \times 10^{4}$ cells $/ \mathrm{cm}^{2}$ and incubated for 24 hours in standard media. Following this media was replaced with complete adipogenic differ-

entiation media (Gibco) and incubated at $37^{\circ} \mathrm{C}$ in a humidified atmosphere of $5 \% \mathrm{CO}_{2}$ for 14 days. Media was changed every 3 days. After 14 days cells were stained for cytoplasmic lipid vacuoles by Oil red $\mathrm{O}$.

\section{Results}

\subsection{Isolation of Human CB-MSC}

After digestion with collagenase I and dispase it was noted that there was minimal to no cells or cellular debris remaining on the bone fragments (Figure 1). In initial experiments the number of cells retrieved from each piece of $2 \times 2 \mathrm{~cm}$ bone yielded $100-1000$ cells per fragments with an average of 400 cells adherent to plastic $(n=9)$. Adherent cells appeared spindle-shaped with long processes (Figure 2).

\subsection{CB-MSC Demonstrate CFU}

The number of cell colonies formed was counted at P0 after seeding cells at different densities $/ \mathrm{cm}^{2}$. CB-MSC demonstrated a statistically significant increase in colony-forming units (CFU) frequency with increasing cell-seeding densities (Table 1). 


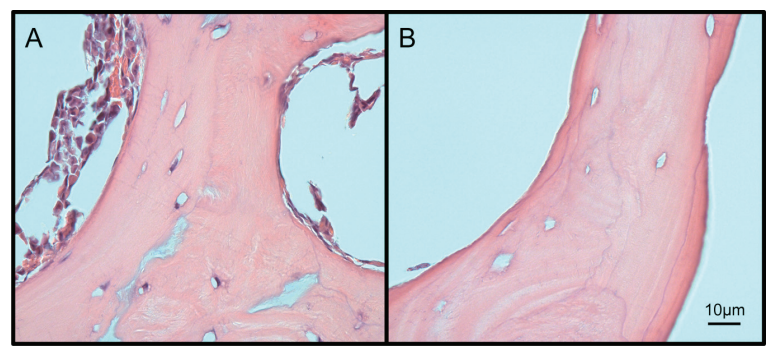

Figure 1 Digestion of bone fragments demonstrates complete removal of cells. Bone fragments were digested using a mixture of Collagenase I and Dispase. Representative picture of bone fragments before digestion (A) and after digestion (B) are shown.

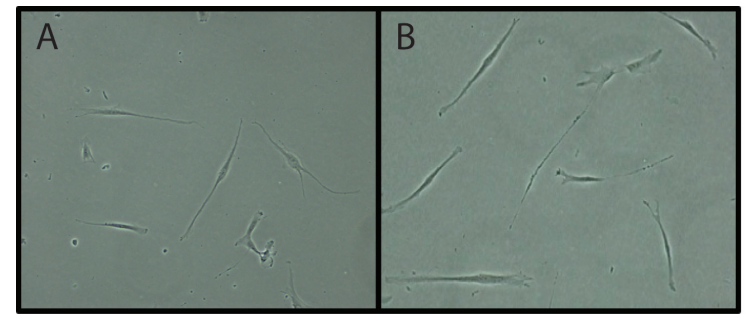

Figure 2 Cell Morphology. Representative image of mesenchymal stem cells (MSC) adherence to plastic and characteristic spindle like morphology following digestion. Cells before (A) and after (B) fluorescence activated assisted cell sorting (FACS) are shown. No difference is seen between the two population's morphology.

Table 1 Markers for fluorescence activated assisted cell sorting (FACS) were chosen on the basis of criteria set forth by the International Society for Cellular Therapy and corroborated in the literature as reliable and specific for the isolation of mesenchymal stromal cells

\begin{tabular}{ll}
\hline \multicolumn{2}{l}{ Markers for Fluorescence Activated Assisted Cell Sorting (FACS) } \\
\hline CD $34^{1}$ & Cluster of differentiation \\
CD $45^{1}$ & Hematopoietic cell marker \\
HLA-DR $^{2}$ & MHC Class II \\
CD $19^{1}$ & B-lymphocyte antigen \\
CD $105^{1}$ & TGF-beta complex \\
CD $73^{1}$ & MSC marker \\
CD $29^{1}$ & MSC antigen marker \\
CD $44^{1}$ & Adhesion molecule \\
CD $90^{1}$ & Thy- $1^{6}$ \\
\hline
\end{tabular}

${ }^{1}$ Cluster differentiation.

${ }^{2}$ Human Leukocyte Antigen - DR locus.

${ }^{3}$ Major histocompatibility complex.

${ }^{4}$ Transforming growth factor.

${ }^{5}$ Mesenchymal stem cell.

${ }^{6}$ Thymocyte antigen-1. 


\subsection{Fluorescence Assisted Cell-Sorting Analysis}

We examined the surface epitopes of the isolated adherent cells in passages $2-4$. Antibodies were selected on the basis of their validation as markers used in the identification of MSC from previously published reports [13]. Comparison of side scatter plot to forward scatter plot demonstrates and homogeneous cell population was obtained (Figure 3A). Cells were strongly positive for CD105 (endoglin, SH2), CD29, CD44, CD 73, and CD90 (Thy-1) (Figure 3B-F). Cells were also stained for lineage markers to ensure that no surface epitopes related to lineage commitment were present. Cells stained negative for CD34, CD45, HLA-DR, and CD19 (Figure 3G-J). The cells were stained in combination utilizing multicolor analysis to demonstrate that cells co-express multiple markers. In all cases there was a small population (5-10\%) of lineage-committed cells. These cells were sorted out leaving a purified cell population with epitopes positive for CD105, CD44, CD29, CD90, and CD73; and negative for CD34, CD45, HLA-DR, and CD19.

Subsequent experiments involve only the use of this isolated cell population.

\subsection{Isolated CB-MSC Demonstrate Tri-Lineage Differentiation Potential}

The biologic property that most uniquely identifies MSC is their capacity for tri-lineage differentiation. That is, differentiation into fat, bone, and cartilage. Following established protocols for differentiation and histological assessment we induced cells into osteoblasts, adipocytes, and chondrocytes.

To demonstrate adipogenic differentiation, cells were cultured in adipogenic media or standard media for 14 days and stained by Oil Red $\mathrm{O}$ stain. Cells cultured in adipogenic media showed formation of lipid vacuoles in the cytoplasm, that stained positively when treated with Oil red $\mathrm{O}$ (Figure 4A, D).

Differentiation of MSC into the osteoblastic lineage was done by stimulating cells in osteogenic media for 14 days and demonstrated polygonal morphology while in induction culture. Following the 14-day incubation period the cells were stained by Von Kossa for mineralization (Figure 4C, F). When compared to cells grown in standard media for the same length of time there was a significantly higher amount of calcium deposition in the osteogenic group.

Lastly, cells were differentiated in a micromass culture for 21 days in chondrogenic inductive media. Cells were also cultured in standard media for the 

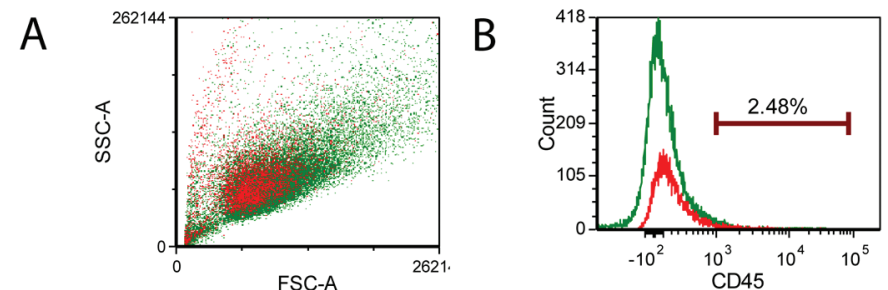

C

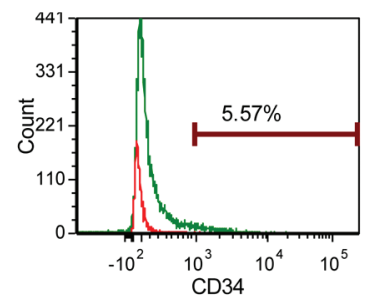

$\mathrm{D}$

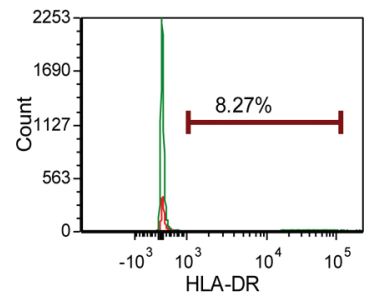

E

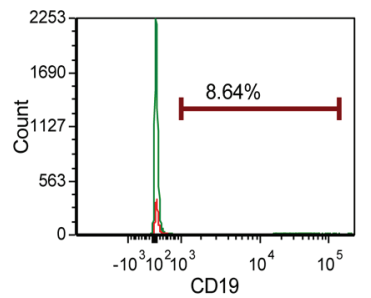

$\mathrm{F}$

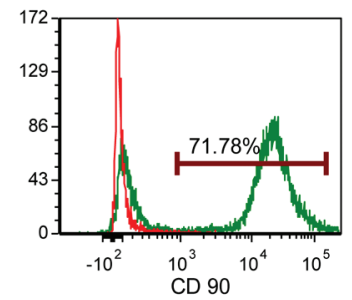

G

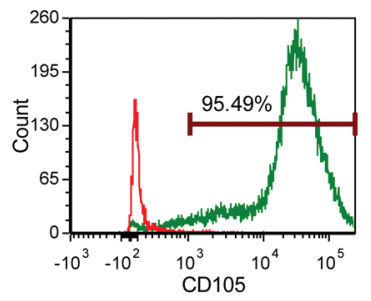

$\mathrm{H}$

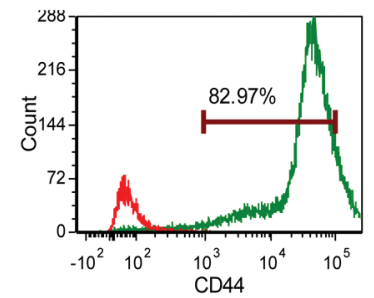

$J$

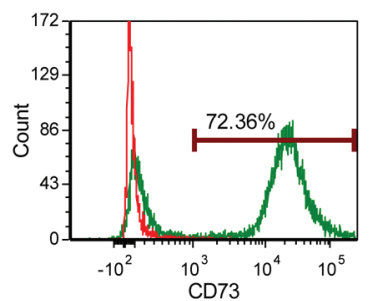

K

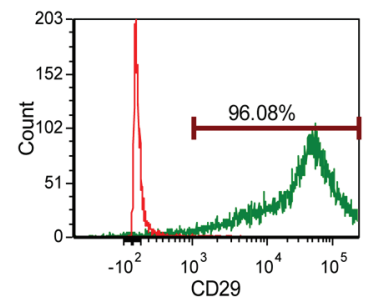

Figure 3 Fluorescence assisted cells sorting of adherent cells. Cells adherent to plastic following processing were found to be uniquely uniform in their cell surface characterization. Following sorting the population of CD34(-) ${ }^{1}$, CD45(-), CD29(+), CD73(+), CD105(+), CD44(+), CD19(-), HLA-DR $(-)^{2}$, and CD90(+) cells were returned to culture for subsequent characterization.

${ }^{1}$ Cluster differentiation factor 34 .

${ }^{2}$ Human Leukocyte Antigen-DR locus. 


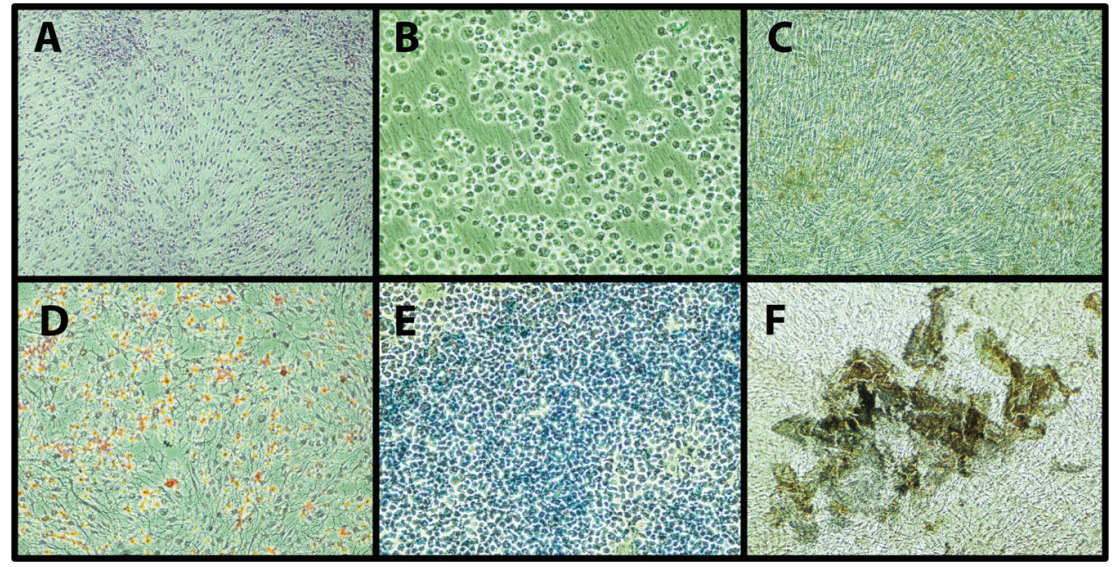

Figure 4 Cells isolated from spinal lamina are capable of osteogenic, chondrogenic, and adipogenic differentiation but not spontaneous differentiation. (A-F) Oil red $\mathrm{O}$ staining of cells cultured in standard complete media (A) or adipogenic media (D) for 14 days. Alcian Blue staining of cells cultured in standard complete media (B) or chondrogenic media (E) for 21 days. Von Kossa staining of isolated cells cultured in standard complete media (C) or osteogenic media (F) for 14 days. Scale bar represents $0.1 \mathrm{~mm}$. All pictures are at $10 \times$ magnification.

21 days as well. Following the incubation time cells were stained for the products of chondrocytic cells, glucosaminoglycans and mucopolysaccharides, by Alcian blue staining. Alcian blue stains demonstrated the appropriate production of cartilage substance by cells induced in chondrogenic differentiation media (Figure 4B, E).

\subsection{Discussion}

Current spine and orthopaedic surgical interventions are focused upon downstream reconstruction and mechanical replacement of musculoskeletal tissues. The future - upstream-will likely be aimed at prevention and/or repair of degenerated tissues, or their replacement with autogenic tissue engineered parts. This 'biological shift' in therapeutics will be founded upon several key ingredients, the most important of which is MSC. To date, a spinal tissue source of cells has yet to be fully characterized. Herein, we describe the isolation and characterization of a tissue specific cell source of MSC.

In this paper, we characterize a novel source of MSC from a previously undescribed source, the adult human cortical bone. The findings demonstrate that a population of cells exists within bone that is not only capable of trilineage differentiation but also carries a cell surface phenotype consistent 
with previous reports of MSC $[12,13]$. As a functional demonstration, when CB-MSCs were seeded at different densities they were able to form clones with frequency that increased with the cell-seeding density. This suggests that paracrine signaling between compact bone-derived cells at P0 occur and may potentiate CFU-F formation in primary culture, as previously reported for MSCs obtained from other sources [14-16]. This study builds on previous studies isolating cells from the spine in that a comprehensive characterization of the cortical bone MSC had never been performed. Further, the method of isolation employed has great efficiency in extracting those cells from human cortical bone to yield a pure population as seen by the homogeneity of the cell population by FACS. This characterization comprehensively demonstrates that the cell isolated from human spinal lamina are, in fact, mesenchymal stem cells.

Using guidelines set forth by the International Society for Cellular Therapy, the cells isolated satisfy all criteria (for MSC) in addition to established criteria for CFU-F formation [13]. Cortical bone has attractive potential as a cell source given the potential ability to harvest in a minimally invasive manner, intraoperatively as part of a spinal procedure, or in the outpatient setting without significant mechanical or biological impact. Newer techniques of more rapid cellular expansion can provide an MSC population ready for immediate surgical implantation in the same setting; or for storage and future surgical use; or for use in autogenic tissue engineering.

An inherent limitation to our study is the population age heterogeneity. A bimodal distribution was seen with most patients undergoing going spinal laminectomy being greater than 60 years old or less than 45 years old. A closer evaluation of age matched samples may yield information regarding the number of CB-MSC able to be isolated as it is presumed that with aging the resident population of stem cells decreases in number. This could also be true for compact bone as a source.

\subsection{Conclusion}

In conclusion, we have described a method for the isolation and characterization of MSC from compact bone and shown that within cortical bone there exists a pure population of CB-MSC. The ability to obtain MSC from this source has advantages over other currently used sources. First, bone can be readily accessed from common sources such as the iliac crest, fractures during fixation (including provisional external fixation), and the spine (laminectomy, as used in this study). The expanded cells can then be used for fracture repair 
or spinal fusion or regenerative efforts (degenerative disc disease). Second, the yield appears to be greater than that found using current bone marrow and fat sources. Lastly, further testing of these cells suggests that MSC obtained using the novel methodology described here produce MSC more abundantly and with greater osteogenic potential/activity than those obtained from the other sources [11].

\section{References}

[1] Yu, D. A., J. Han, and B. S. Kim, Stimulation of Chondrogenic Differentiation of Mesenchymal Stem Cells. 2012. Int. J. Stem Cells 5: $16-22$.

[2] Scott, M. A., et al. 2011. Current methods of adipogenic differentiation of mesenchymal stem cells. Stem Cells Dev. 20: 1793-1804.

[3] Maxson, S., et al. 2012. Concise review: role of mesenchymal stem cells in wound repair. Stem Cells Translat. Med. 1: 142-149.

[4] Cashman, T. J., V. Gouon-Evans, and K. D. Costa. 2013. Mesenchymal stem cells for cardiac therapy: practical challenges and potential mechanisms. Stem Cell Rev. 9: 254-265.

[5] Ringe, J., et al. 2008. Human mastoid periosteum-derived stem cells: promising candidates for skeletal tissue engineering. J. Tissue Eng. Regen. Med. 2: 136-146.

[6] Lyahyai, J., et al. 2012. Isolation and characterization of ovine mesenchymal stem cells derived from peripheral blood. BMC Vet. Res. 8: 169.

[7] Reynolds, J. A., et al. 2013. Improving cardiovascular outcomes in rheumatic diseases: therapeutic potential of circulating endothelial progenitor cells. Pharmacol. Therapeut.

[8] Gullo, F. and C. De Bari. 2013. Prospective purification of a subpopulation of human synovial mesenchymal stem cells with enhanced chondro-osteogenic potency. Rheumatology 52: 1758-1768.

[9] Mendez-Ferrer, S., et al. 2010. Mesenchymal and haematopoietic stem cells form a unique bone marrow niche. Nature 466: 829-834.

[10] Hicok, K. C., et al. 2004. Human adipose-derived adult stem cells produce osteoid in vivo. Tissue Eng. 10: 371-380.

[11] Fernandez-Moure, J. S., et al. 2015. Enhanced osteogenic potential of mesenchymal stem cells from cortical bone: a comparative analysis. Stem Cell Res. Ther. 6: 203. 
[12] Zhu, H., et al. 2010. A protocol for isolation and culture of mesenchymal stem cells from mouse compact bone. Nat. Protoc. 5: 550-560.

[13] Dominici, M., et al. 2006. Minimal criteria for defining multipotent mesenchymal stromal cells. The International Society for Cellular Therapy position statement. Cytotherapy 8: 315-317.

[14] Corradetti, B., et al. 2013. Mesenchymal stem cells from amnion and amniotic fluid in the bovine. Reproduction 145: 391-400.

[15] Lange-Consiglio, A., et al. 2012. Characterization and potential applications of progenitor-like cells isolated from horse amniotic membrane. J. Tissue Eng. Regen. Med. 6: 622-635.

[16] Lovati, A. B., et al. 2001. Characterization and differentiation of equine tendon-derived progenitor cells. J. Biol. Regul. Homeostat. Agents 25: S75-S84.

\section{Biographies}

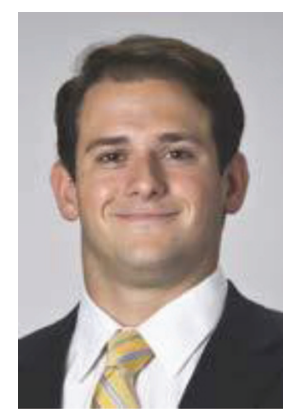

J. S. Fernandez-Moure M.D., M.S. is Chief Administrative Resident in Surgery at Houston Methodist Hospital in Houston, Texas. He graduated from college at Middlebury College in Middlebury, Vermont with degrees in Biochemistry and Spanish in 2002. Following this Dr. Fernandez-Moure went onto to the Rutgers University Graduate School of Biomedical Sciences in Newark, New Jersey to begin his Master's in Science with a focus in molecular biology and stem cell biology. During this time he also enrolled in Rutgers New Jersey Medical School where he obtained his M.D. degree. Following this Dr. Fernandez-Moure was accepted into the residency in general surgery at Houston Methodist Hospital. Dr. Fernandez-Moure became the American Board of Surgery's first surgeon scientist fellow and during that time was promoted to Instructor in the Department of Nanomedicine. 


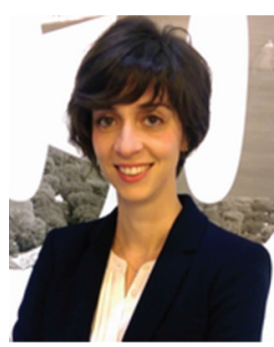

B. Corradetti Ph.D., M.Sc. is currently an assistant professor at Universita Politecnica Delle Marche, Department of Life and Environmental Sciences in Ancona, Italy.

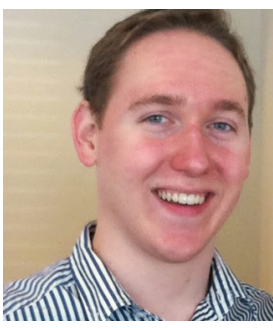

T. Janecek, Houston Methodist. Leading Medicine. U.S. News \& World Report has recognized Houston Methodist as one of the nation's top 20 hospitals, placing it for the second time on the magazine's prestigious Honor Roll. It is also designated as a Magnet hospital for excellence in nursing.

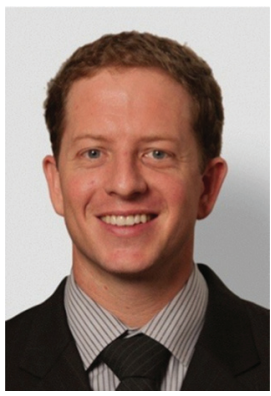

J. V. Eps M.D. is a senior resident physician in General Surgery at Houston Methodist Hospital and a Research Associate in the Center for Regenerative and Biomimetic Medicine at the Houston Methodist Research Institute. 
Dr. Van Eps received his bachelor's degree from Azusa Pacific University in southern California and his M.D. degree from Saint Louis University School of Medicine. He plans to pursue a career in academic subspecialty surgery and his research interests include unique applications of advanced biomaterial matrices, controlled release mechanisms and autologous/synthetic factors as they relate to: augmentation of surgical wound healing, surgical analgesia, surgical device development, osteogenic and chondrogenic applications, and stem cell biology.

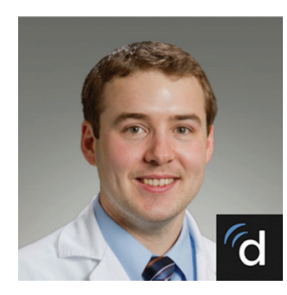

M. Burn M.D. is a Chief Resident in Orthopedic Surgery at Houston Methodist Hospital in Houston TX. Dr. Burns is a graduate of LSU School of Medicine in Shreveport.

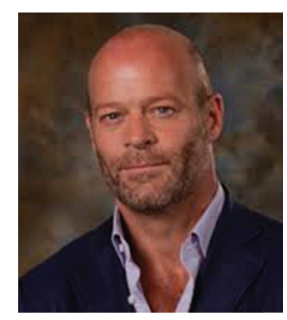

B. K. Weiner is the Vice Chairman of Orthopaedic Surgery and the Chief of Spine Surgery at Houston Methodist. He has been at Houston Methodist since 2006 and serves as the Director for the Orthopaedic Surgery Residency Program. Dr. Weiner specializes in complex surgeries of the cervical, thoracic, and lumbar spines and he is recognized as a leader in spinal microsurgery. 


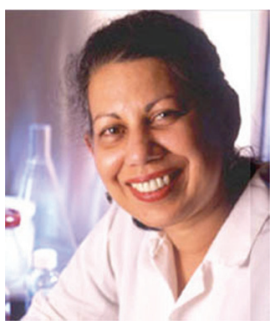

P. Rameshwar obtained her education at the University of WisconsinMadison, WI, USA and Rutger's University, Newark, New Jersey, USA. Her main academic appointment is at Rutgers-New Jersey Medical School, Department of Medicine-Hematology/Oncology. Professor Rameshwar also has an appointment at the Graduate School of Biomedical Sciences. She is also a member of the joint doctoral program in biomedical engineering between University of Medicine and Dentistry of New Jersey and New Jersey Institute of New Jersey.

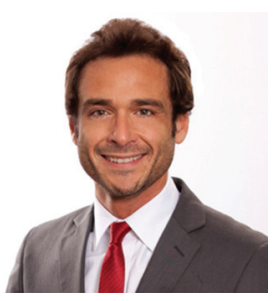

E. Tasciotti Ph.D. is associate professor of Nanomedicine in the Institute for Academic Medicine at Houston Methodist Hospital in Houston, TX. Dr. Tasciotti earned his B.S. in Biological Sciences and his M.S. in Molecular Biology at Scuola Normale Superiore Pisa, Italy and a Ph.D. in Molecular Medicine from a joint program of the Scuola Normale Superiore Pisa with the International Center for Biotechnology and Genetic Engineering (ICGEB) in 2004. Dr. Tasciotti research as a Ph.D. student focused on gene and stem cell therapy of cancer. He moved to the United States in 2006 as a senior postdoctoral fellow in the Department of Nanomedicine and Biomedical Engineering at the University of Texas Health Science Center at Houston. Following this appointment he transitioned to his current role of Director for the Center for Biomitmetic Medicine at Houston Methodist Hospital. 
